\title{
Cerebellar liponeurocytoma:An updated follow-up of a case presenting histopathological and clinically aggressive features
}

\author{
Gallina Pasquale, Buccoliero Anna M aria', Pirillo Vania, Pansini Gastone, Di Lorenzo N icola \\ Departments of Neurosurgery and ${ }^{1}$ Human Pathology, University of Florence, Florence, Italy
}

\begin{tabular}{l} 
\\
\hline Address for correspondence: \\
Dr. Pasquale Gallina, \\
Department of Neurosurgery, \\
University of Florence, \\
CTO Hospital, 1, Largo P. Palagi \\
50139 - Florence, Italy. \\
E-mail: pgal@ unifi.it
\end{tabular}

DOI: $10.4103 / 0028-3886.51294$

\begin{abstract}
Abstrat
Cerebellar liponeurocytoma is a rare tumor recently included by World Health Organization in the classification of brain tumors as a separate clinicopathological entity separate from medulloblastoma. However, because of the rarity of the tumor, the natural history of the tumor is still not yet been defined. We report a patient with cerebellar liponeurocytoma with unusual clinical and pathological aggressive features. This patient suggests the possible existence of different histological grades of liponeurocytoma and its possible progression to a malignant grade.
\end{abstract}

Key words: Cerebellar liponeurocytoma, intraoperative radiation therapy, recurrence

\section{Introduction}

Cerebellar liponeurocytoma is a distinct, newly identified, rare tumor and occurs in adults. It is a relatively benign lesion and can be classified as Grade I or Grade II tumor of the World Health Organization (WHO) classification. ${ }^{[1,2]}$ Gross total removal with possible additional External Beam Radiation Therapy (EBRT) is the treatment of choice. ${ }^{[3]}$ Because of the rarity of the tumor, the natural history of cerebellar liponeurocytoma has not yet been defined and the benign nature of the tumor is being questioned. ${ }^{[4]}$ We had documented this patient with cerebellar liponeurocytoma earlier. At that time he had the total removal of the tumor without additional therapy ${ }^{[5]}$ During the follow-up he had early recurrence with histological features of malignant progression. This prompted us to report the updated follow-up of the patient.

\section{Case Report}

This 64-year-old male patient had undergone complete and uncomplicated surgical excision of a right cerebellar liponeurocytoma, via lateral sub-occipital craniotomy. ${ }^{[5]}$
On histology the lesion showed occasional mitoses and a high proliferation rate (MIB-1 immunostaining: 15\% in more positive areas ${ }^{[6]}$ [Figure 1]. No supplementary treatment was followed. At the six-monthly clinical checks, the patient was asymptomatic and annual magnetic resonance imaging (MRI) showed no evidence of contrast enhancement attributable to a tumor. Three and half years after the diagnosis of the initial lesion, follow-up MRI showed tumor recurrence. Patient had no neurological deficits. The patient underwent a second surgery. Reopening of the previous craniotomy allowed gross total resection of the lesion. As intraoperative histopathological examination of the specimen confirmed the diagnosis of liponeurocytoma recurrence, intraoperative radiation therapy (IORT) was given, using a miniaturized X-ray device (Photon Radiosurgery System, PRS 400; Carl Zeiss, Oberkochen, Germany) and a radiation dose of 15.4 Gy was delivered to the tumor bed, at $5 \mathrm{~mm}$ depth from the surface of the surgical cavity, for $25 \mathrm{~min}$. The post-treatment course was uneventful and the patient was discharged five days after the procedure. No additional therapy was suggested and only close follow-up examinations were scheduled. At four years follow-up from the second 
operation, the patient was asymptomatic and MRI showed no tumor recurrence.

\section{Pathological findings}

Similar to the lesion in the first presentation, the recurrence showed two different cellular components, poorly differentiated isomorphic small closely packed cells and mature adipocyte-like cells. ${ }^{[7]}$ The small cells had round to oval hyperchromatic nuclei and small cytoplasms. Some lipid-laden cells were sparse while others were arranged in groups. The majority of them showed a single fat vacuole, while the rest showed several small vacuoles pressing the nuclei to the cell periphery. Homer-Wright rosettes and pseudorosettes were observed in the recurrence. Vascular hyperplasia and areas of micro-necrosis [Figure 2a] and micro-hemorrhage were more obvious in the recurrence with respect to the

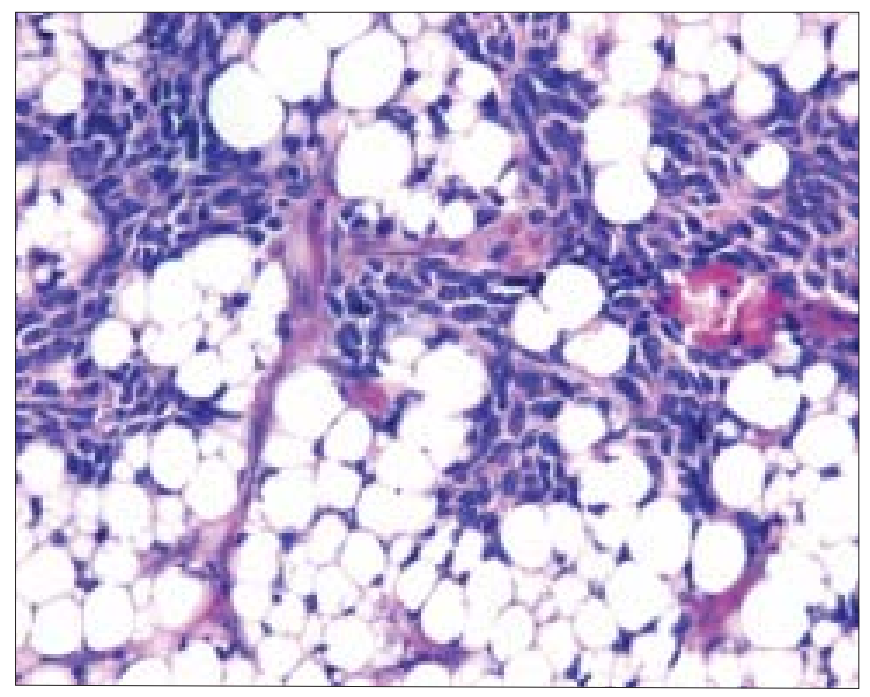

Figure 1: First presentation of right cerebellar liponeurocytoma: Small, closely packed cells and mature adipocyte-like cells. Original magnification: $\times 200$

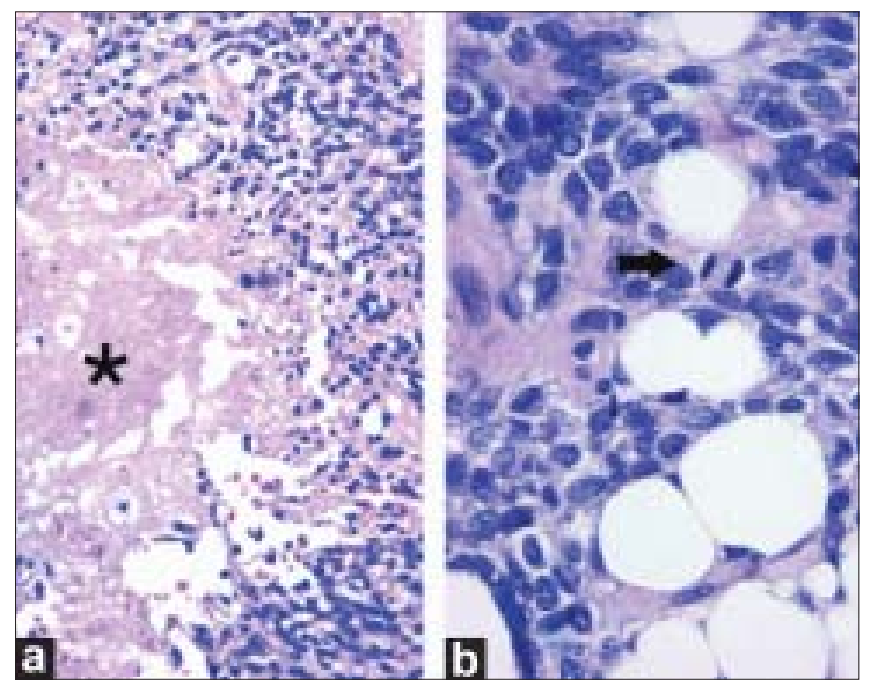

Figure 2: Recurrence: Necrosis (asterisk, a) and mitosis (arrow, b). Original magnification: $\times 100(a) ; \times 200(b)$ first presentation. Analogously, mitoses and proliferation index as evaluated by MIB-1 antibody, respectively increased from rare to 2-3 [Figure 2b] and from 15\% to $20 \%$. Areas of micro-necrosis, micro-hemorrhage and moderate vascular hyperplasia were observed in the relapse. Infiltrating neoplastic cells were visible in the perilesional tissue [Figure 3].

\section{Discussion}

Cerebellar liponeurocytoma, characteristically showing divergent glio-neuronal differentiation and lipidized neoplastic cells and having a much more benign clinical outcome than the more common medulloblastoma, has been considered an adult lipomatous variant of the medulloblastoma. ${ }^{[8-10]}$ However, recent genetic studies have clarified that liponeurocytoma could be a distinct entity, unrelated to medulloblastoma. ${ }^{[11]}$ Based on literature reviews, recurrence has been observed in $20-32 \%$ of patients, on an average, 10 years after surgery (range 8-12 years). ${ }^{[3,12]}$ Total resection is considered the optimal treatment, while additional EBRT is

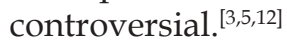

In 2003, Jenkinson ${ }^{[4]}$ reported an unusual case of a cerebellar liponeurocytoma that presented a first recurrence 12 months after subtotal removal followed by EBRT of the first lesion, and a second recurrence three months after a second surgical removal. Despite the absence of atypical histological features in both the primary and the relapsed tumors, apart from leptomeningeal invasion, the unusually aggressive case described by Jenkinson suggested that cerebellar liponeurocytoma may not be as benign as previously thought. Based on this report, more aggressive therapeutic approaches with additional chemotherapy were considered..$^{[13-15]}$ Similar to Jenkinson's case, our patient experienced a rather early recurrence, despite the fact that postoperative MRI confirmed complete resection of the tumor. Differently from Jenkinson's

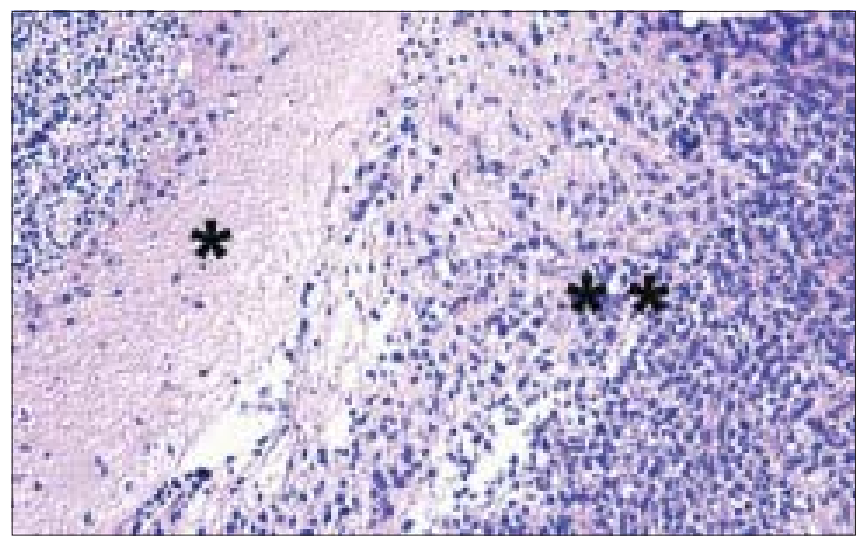

Figure 3: Recurrence: Infiltrating neoplastic cells in the perilesional tissue (asterisk: Cerebellar cortex; double asterisk infiltrating tumoral cells) Original magnification: $\times 100$ 
case, our patient presented histopathological signs of an aggressive form of liponeurocytoma in both the first presentation and the recurrence (mitoses, pleomorphism, moderate endothelial proliferation and high proliferation rate). Based on these morphological findings, it was suggested by our group that the increased presence of mitosis and a MIB-1 cells positivity by over $10 \%$ of the total neoplastic cells, may indicate a malignant potential lesion. ${ }^{[16]}$ Moreover, a few differences were observed between the recurrence and the primary lesion: Vascular hyperplasia, areas of micro-necrosis and micro-hemorrhage, mitoses, synaptic vesicles and MIB-1 positive neoplastic cells were more obvious in the recurrence with respect to the first lesion. These results indicate a malignant progression of the liponeurocytoma. ${ }^{[7,17,18]}$

Hence, the prognosis of cerebellar liponeurocytoma appears less predictable and its management more debatable, also considering the recent description of very exceptional supratentorial intraventricular cases. ${ }^{[19]}$ Taking into account the expected favorable course of cerebellar liponeurocytoma and the lack of evidence for the usefulness of postoperative radiation therapy, EBRT was not delivered after the first surgery in this patient. In the presence of this unexpected and early recurrence, we performed a second surgery followed by IORT of the surgical cavity. At our institution, IORT is delivered using PRS, a miniature radiation source that emits low-energy X-ray photons. ${ }^{[17]}$ Considering the histological features and the presence of infiltrating neoplastic cells into perilesional tissue, the aim of IORT was to increase local control, taking advantage of the radiobiological effect of the delivery, in a single session, of a high dose of radiation into the tumor bed. This would thereby eliminate residual tumor cells which are the hypothetical source of recurrence. EBRT was not given, reserving such an option for the possibility of further recurrence. Chemotherapy was not recommended due to the lack of convincing usefulness and in light of recent studies that showed liponeurocytoma as a distinct entity from medulloblastoma. ${ }^{[11]}$ The follow-up in our case is too short a period and the natural history of cerebellar liponeurocytoma is too ambiguous for predictions on its clinical course. Close follow-up examinations were scheduled in order to detect any possible recurrence early on.

In conclusion, our case confirmed the existence of different grades of malignancy among liponeurocytomas. Large surgical resection is recommended. IORT may be an additional tool to increase local control and thus may prevent early recurrence.

\section{References}

1. Bechtel JT, Patton JM, Takei Y. Mixed mesenchymal and neuroectodermal tumor of the cerebellum. Acta Neuropathol (Berl) 1978;41:261-3.

2. Kleihues P, Cavenee WK. Pathology and geneties of tumours of the nervous system. (World Health Organization Classification of Tumors). Lyon, France: International Agency for Research on Cancer (IARC); 2000.

3. Jackson TR, Regine WF, Wilson D, Davis DG. Cerebellar liponeurocytoma: Case report and review of the literature. J Neurosurg 2001;95:700-3.

4. Jenkinson MD, Bosma JJ, Du Plessis D, Ohgaki H, Kleihues P, Warnke P, et al. Cerebellar liponeurocytoma with an unusually aggressive clinical course: Case report. Neurosurgery 2003;53:1425-8.

5. Cacciola F, Conti R, Taddei GL, Buccoliero AM, Di Lorenzo N. Cerebellar liponeurocytoma: Case report with considerations on prognosis and management. Acta Neurochir (Wien) 2002;144:829-33.

6. Taddei GL, Buccoliero AM, Caldarella. Cerebellar liponeurocytoma: Immunohistochemical and ultrastructural study of a case. Ultrastruct Pathol 2001;25:59-63.

7. Buccoliero AM, Caldarella A, Bacci S, Gallina P, Taddei A, Di Lorenzo N, et al. Cerebellar liponeurocytoma: Morphological, immunohistochemical and ultrastructural study of a relapsed case. Neuropathology 2005;25:77-83.

8. Akhaddar A, Zrara I, Gazzaz M, El Moustarchid B, Benomar S, Boucetta M. Cerebellar liponeurocytoma (lipomatous medulloblastoma). J Neuroradiol 2003;30:121-6.

9. Owler BK, Makeham JM, Shingde M, Besser M. Cerebellar liponeurocytoma. J Clin Neurosci 2005;12:326-9.

10. Tatke M, Singh AK. Cerebellar liponeurocytoma: A case report. Indian J Pathol Microbiol 2005;48:29-31.

11. Horstmann S, Perry A, Reifenberger G. Genetic and expression profiles of cerebellar liponeurocytomas. Brain Pathol 2004;14:281-9.

12. Valery CA, Sakka LJ, Poirier J. Problematic differential diagnosis between cerebellar liponeurocytoma and anaplastic oligodendroglioma. Br J Neurosurg 2004;18:300-3.

13. Rock JP; Comment in Jenkinson et al. Cerebellar liponeurocytoma with an unusually aggressive clinical course: Case report. Neurosurgery 2003;53:1428.

14. Seifert V, Jadik S; Comment in Jenkinson et al. Cerebellar liponeurocytoma with an unusually aggressive clinical course: Case report. Neurosurgery 2003;53:1428.

15. Westhpal M; Comment in Jenkinson et al. Cerebellar liponeurocytoma with an unusually aggressive clinical course: Case report. Neurosurgery 2003;53:1428.

16. Bechtel.JT, Patton JM, Takei Y. Mixed mesenchymal and neuroectodermal tumor of the cerebellum. Acta Neuropathol (Berl) 1978;41:261-3.

17. Gallina P, Francescon P, Cavedon C, Casamassima F, Mungai R, Terrin G, et al. Stereotactic interstitial radiosurgery and intraoperative radiotherapy with a miniature x-ray device in the treatment of selected brain tumors. Preliminary results. In: Kondiziolka D, Mc Dermott M, Regis L, Smee R, Takakura K, editors. Radiosurgery: Basel, Karger: Vol 4, 2002, p. 167-78.

18. Aker FV, Ozkara S, Eren P, Peker O, ArmaÄ̈̈an S, Hakan T. Cerebellar liponeurocytoma / lipidized medulloblastoma. J Neurooncol 2005;71:53-9.

19. Kuchelmeister K, Nestler U, Siekmann R, Schachenmayr W. Liponeurocytoma of the left lateral ventricle-case report and review of the literature. Clin Neuropathol 2006;25:86-94.

Accepted on 06-03-2009

Source of Support: Nil, Conflict of Interest: None declared. 